\title{
SOLUTION OF NONLINEAR VOLTERRA-HAMMERSTEIN INTEGRAL EQUATIONS VIA SINGLE-TERM WALSH SERIES METHOD
}

\author{
B. SEPEHRIAN AND M. RAZZAGHI
}

Received 5 November 2004

Single-term Walsh series are developed to approximate the solutions of nonlinear Volterra-Hammerstein integral equations. Properties of single-term Walsh series are presented and are utilized to reduce the computation of integral equations to some algebraic equations. The method is computationally attractive, and applications are demonstrated through illustrative examples.

\section{Introduction}

Several numerical methods for approximating the solution of Hammerstein integral equations are known. For Fredholm-Hammerstein integral equations, the classical method of successive approximations was introduced in [16]. A variation of the Nystrom method was presented in [11]. A collocation-type method was developed in [9]. In [3], Brunner applied a collocation-type method to nonlinear Volterra-Hammerstein integral equations and integro-differential equations, and discussed its connection with the iterated collocation method. Han [6] introduced and discussed the asymptotic error expansion of a collocation-type method for Volterra-Hammerstein integral equations. The methods in $[6,9]$ transform a given integral equation into a system of nonlinear equations, which has to be solved with some kind of iterative method. In [9], the definite integrals involved in the solution may be evaluated analytically only in favorable cases, while in [6] the integrals involved in the solution have to be evaluated at each time step of the iteration.

Orthogonal functions, often used to represent arbitrary time functions, have received considerable attention in dealing with various problems of dynamic systems. The main characteristic of this technique is that it reduces these problems to those of solving a system of algebraic equations, thus greatly simplifying the problem. The approach is based on converting the underlying differential equations into integral equations through integration, approximating various signals involved in the equations by truncated orthogonal series and using the operational matrix of integration $P$ to eliminate the integral operations. The matrix $P$ can be uniquely determined based on the particular orthogonal functions. Orthogonal functions have also been proposed to solve linear integral 
equations. Hsiao and Chen [7] introduced the Walsh series operational matrix of integration to solve linear integral equations. Due to the nature of the Walsh functions, the solutions obtained were piecewise constant. Moreover, Hwang and Shih [8], Chang and Wang [4], Chou and Horng [5], and Razzaghi et al. [14] used the operational matrices of integration associated with Laguerre polynomials, Legendre polynomials, Chebyshev polynomials, and Fourier series to derive continuous solutions for linear integral equations.

Walsh functions (WF) have found wide applications in signal processing, communication, and pattern recognition [13]. Rao et al. [12] presented a method of extending computation beyond the limit of the initial normal interval in Walsh series analysis of dynamical systems. In the last method, various time functions in the system were first expanded as truncated WF with unknown coefficients. Then, by using the Kronecker product [10], the unknown coefficient of the rate variable was obtained by finding the inverse of a square matrix. It was shown that this method involves some numerical troubles if the dimension of this matrix is large. Rao et al. [12] introduced single-term Walsh series (STWS) to remove the inconveniences in WF technique. Furthermore, Balachandran and Murugesan [1,2] applied STWS technique to the analysis of linear and nonlinear singular systems.

In the present article we are concerned with the application of STWS to the numerical solution of nonlinear Volterra-Hammerstein integral equations of the form

$$
y(t)=f(t)+\int_{0}^{t} \kappa(t, s) g(s, y(s)) d s, \quad 0 \leq t<1
$$

where $f, g$, and $\kappa$ are given continuous functions, with $g(s, y)$ nonlinear in $y$. We assume that (1.1) has a unique solution $y$ to be determined. The method presented here does not require the Kronecker product of matrices and there is no need for the operational matrix of integration. One main merit of using this technique is that it provides pointwise, Block-pulse, and continuous approximations for solution of (1.1).

The paper is organized as follows. Section 2 is devoted to the basic formulation of WF and STWS required for our subsequent development. In Section 3, the solution of (1.1) using STWS is considered. Section 4 is devoted to two numerical examples. In both numerical examples we demonstrate the accuracy of the proposed scheme by comparing our numerical finding with the exact solutions.

\section{Properties of WF and STWS}

2.1. Walsh functions. A function $h(t)$, integrable in $[0,1)$, may be approximated using WF as

$$
h(t)=\sum_{i=0}^{\infty} h_{i} \phi_{i}(t),
$$

where $\phi_{i}(t)$ is the $i$ th WF and $h_{i}$ is the corresponding coefficient. In practice, only the first $m$ terms of the WF are considered, where $m$ is an integral power of 2. Then from (2.1) 
we get

$$
h(t)=\sum_{i=0}^{m-1} h_{i} \phi_{i}(t)=H^{T} \Phi(t),
$$

where

$$
\begin{gathered}
H=\left(h_{0}, h_{1}, \ldots, h_{m-1}\right)^{T}, \\
\Phi(t)=\left(\phi_{0}(t), \phi_{1}(t), \ldots, \phi_{m-1}(t)\right)^{T} .
\end{gathered}
$$

The coefficients $h_{i}$ are chosen to minimize the mean integral square error

$$
\epsilon=\int_{0}^{1}\left(h(t)-H^{T} \Phi(t)\right)^{2} d t
$$

and are given by

$$
h_{i}=\int_{0}^{1} h(t) \phi_{i}(t) d t
$$

The integration of the vector $\Phi(t)$ defined in (2.3) can be approximated by

$$
\int_{0}^{t} \Phi\left(t^{\prime}\right) d t^{\prime} \simeq E \Phi(t)
$$

where $E_{m \times m}$ is the $m \times m$ operational matrix for integration with the element $E_{1 \times 1}=1 / 2$ and is given in [7].

2.2. Single-term Walsh series. With the STWS approach, in the first interval, the given function is expanded as single-term Walsh series in the normalized interval $\tau \in[0,1)$, which corresponds to $t \in[0,1 / m)$, by defining $\tau=m t$, with $m$ being any integer. In STWS, the matrix $E$ in (2.6) becomes $E=1 / 2$.

Let $\dot{y}(\tau)$ and $y(\tau)$ be expanded by STWS series in the first interval as

$$
\begin{aligned}
& y(\tau)=Y^{(1)} \phi_{0}(\tau), \\
& \dot{y}(\tau)=V^{(1)} \phi_{0}(\tau) .
\end{aligned}
$$

Integrating (2.8) with $E=1 / 2$ we get

$$
Y^{(1)}=\frac{1}{2} V^{(1)}+y(0)
$$

where $y(0)$ is the initial condition. According to Sannuti [15] we have

$$
V^{(1)}=\int_{0}^{1} \dot{y}(\tau) d \tau=y(1)-y(0) .
$$

From (2.9) and (2.10) we get

$$
y(1)=2 Y^{(1)}-y(0)
$$


In general for the $i$ th interval we have

$$
y(i)=2 Y^{(i)}-y(i-1), \quad i=1,2, \ldots, m .
$$

In (2.12) $Y^{(i)}$ and $y(i)$ give the Block-pulse and the discrete values of $y(t)$, respectively.

\section{Solution of nonlinear Volterra-Hammerstein integral equations by STWS}

Consider the Volterra-Hammerstein integral equation (1.1). Let

$$
z(s)=g(s, y(s)), \quad 0 \leq s<1 .
$$

In order to use STWS, we first divide the interval $[0,1)$ into $m$ equal subintervals, where $m$ is any positive integer. We then transform each interval $(i-1) / m \leq s \leq t<i / m, i=$ $1, \ldots, m$, into $[0,1)$ by means of transformations $\tau_{i}=m t-(i-1)$ and $\lambda_{i}=m s-(i-1)$. Using (1.1) and (3.1), in the first interval $\tau_{1}$ we have

$$
y\left(\tau_{1}\right)=f\left(\tau_{1}\right)+\frac{1}{m} \int_{0}^{\tau_{1}} \kappa\left(\tau_{1}, \lambda_{1}\right) z\left(\lambda_{1}\right) d \lambda_{1}
$$

and similarly in the $i$ th interval we have

$$
y\left(\tau_{i}\right)=f\left(\tau_{i}\right)+\frac{1}{m} \sum_{j=1}^{i-1} \int_{0}^{1} \kappa\left(\tau_{i}, \lambda_{j}\right) z\left(\lambda_{j}\right) d \lambda_{j}+\frac{1}{m} \int_{0}^{\tau_{i}} \kappa\left(\tau_{i}, \lambda_{i}\right) z\left(\lambda_{i}\right) d \lambda_{i}
$$

Let $f\left(\tau_{i}\right)$ and $\kappa\left(\tau_{i}, \lambda_{j}\right)$ be expressed by STWS as

$$
\begin{array}{cl}
f\left(\tau_{i}\right)=F^{(i)} \phi_{0}\left(\tau_{i}\right), & i=1, \ldots, m, \\
\kappa\left(\tau_{i}, \lambda_{j}\right)=K^{(i, j)} \phi_{0}\left(\tau_{i}\right) \phi_{0}\left(\lambda_{j}\right), & i=1, \ldots, m, j=1, \ldots, i,
\end{array}
$$

where $K^{(i, j)}$ is obtained from

$$
K^{(i, j)}=\int_{0}^{1} \int_{0}^{1} \kappa\left(\tau_{i}, \lambda_{j}\right) d \tau_{i} d \lambda_{j}=m^{2} \int_{(i-1) / m}^{i / m} \int_{(j-1) / m}^{j / m} \kappa(t, s) d t d s .
$$

Similarly to (2.7), $y(s)$ is expanded by STWS in the $i$ th interval as

$$
y\left(\lambda_{i}\right)=Y^{(i)} \phi_{0}\left(\lambda_{i}\right)
$$

Equations (3.1) and (3.7) give

$$
z\left(\lambda_{i}\right)=g\left(\lambda_{i}, Y^{(i)} \phi_{0}\left(\lambda_{i}\right)\right)
$$

Let $z\left(\lambda_{i}\right)$ be expressed by STWS as

$$
z\left(\lambda_{i}\right)=Z^{(i)} \phi_{0}\left(\lambda_{i}\right)
$$

where $Z^{(i)}$ is in terms of $Y^{(i)}, i=1, \ldots, m$. 
Using (2.6) with $E=1 / 2$, (3.5), and (3.9), we get

$$
\int_{0}^{\tau_{i}} \kappa\left(\tau_{i}, \lambda_{i}\right) z\left(\lambda_{i}\right) d \lambda_{i}=\frac{1}{2} K^{(i, i)} Z^{(i)} \phi_{0}\left(\tau_{i}\right), \quad i=1, \ldots, m
$$

From (2.6), (3.2), and (3.10), the Block-pulse value $Y^{(1)}$ in the first interval is given by

$$
Y^{(1)}=F^{(1)}+\frac{1}{2 m} K^{(1,1)} Z^{(1)}
$$

which is a nonlinear equation for $Y^{(1)}$, and similarly in the ith interval, we have

$$
Y^{(i)}=F^{(i)}+\frac{1}{m} \sum_{j=1}^{i-1} K^{(i, j)} Z^{(j)}+\frac{1}{2 m} K^{(i, i)} Z^{(i)}, \quad i=2, \ldots, m
$$

By solving (3.11) for $Y^{(1)}$ and (3.12) for $Y^{(i)}, i=2, \ldots, m$, we can obtain Block-pulse values of $y(t)$. Then, by using (2.10) discrete approximations of $y(t)$ can be calculated. Also, for $\alpha \in[0,1 / m)$, from (1.1), (3.2), and (3.9) we have

$$
y(\alpha)=f(\alpha)+Z^{(1)} \int_{0}^{\alpha} \kappa(\alpha, s) d s, \quad \alpha \in\left[0, \frac{1}{m}\right)
$$

and for $\alpha \in[(i-1) / m, i / m]$ from (3.3) and (3.9),

$$
\begin{gathered}
y(\alpha)=f(\alpha)+\sum_{j=1}^{i-1} Z^{(j)} \int_{(j-1) / m}^{j / m} \kappa(\alpha, s) d s+Z^{(i)} \int_{(i-1) / m}^{\alpha} \kappa(\alpha, s) d s, \\
\alpha \in\left[\frac{i-1}{m}, \frac{i}{m}\right], i=2, \ldots, m .
\end{gathered}
$$

Equations (3.13) and (3.14) provide continuous approximations for $y(t)$ at every $\alpha \in$ $[0,1)$.

\section{Illustrative examples}

4.1. Example 1. Consider the nonlinear Volterra-Hammerstein integral equation [17]

$$
y(t)=-\frac{15}{56} t^{8}+\frac{13}{14} t^{7}-\frac{11}{10} t^{6}+\frac{9}{20} t^{5}+t^{2}-t+\int_{0}^{t}(t+s) y^{3}(s) d s, \quad t \in[0,1) .
$$

By applying the technique described in the preceding sections, (4.1) is solved. The computational results for discrete approximations (DA) and continuous approximations (CA) of $y(t)$ with $m=20$ and $m=40$, together with the exact solution $y(t)=t^{2}-t$, are given in Table 4.1. In Table 4.2, the least square errors for Block-pulse and continuous 


\section{Nonlinear integral equations via STWS}

Table 4.1. Estimated values of $y(t)$ using STWS with $m=20$ and 40 together with exact values for Example 1.

\begin{tabular}{|c|c|c|c|c|c|}
\hline$t$ & DA with $m=20$ & CA with $m=20$ & DA with $m=40$ & CA with $m=40$ & Exact \\
\hline 0.0 & 0.00000 & 0.00000 & 0.00000 & 0.00000 & 0.0 \\
\hline 0.1 & -0.09000 & -0.09000 & -0.09000 & -0.09000 & -0.09 \\
\hline 0.2 & -0.16000 & -0.16000 & -0.16000 & -0.16000 & -0.16 \\
\hline 0.3 & -0.21000 & -0.20999 & -0.21000 & -0.21000 & -0.21 \\
\hline 0.4 & -0.24000 & -0.23999 & -0.24000 & -0.24000 & -0.24 \\
\hline 0.5 & -0.24999 & -0.24999 & -0.25000 & -0.25000 & -0.25 \\
\hline 0.6 & -0.23998 & -0.23999 & -0.24000 & -0.24000 & -0.24 \\
\hline 0.7 & -0.20997 & -0.20999 & -0.20999 & -0.21000 & -0.21 \\
\hline 0.8 & -0.15997 & -0.15998 & -0.15999 & -0.16000 & -0.16 \\
\hline 0.9 & -0.08997 & -0.08997 & -0.08999 & -0.08999 & -0.09 \\
\hline 0.1 & 0.00003 & 0.00003 & 0.00001 & 0.00001 & 0.0 \\
\hline
\end{tabular}

Table 4.2. Least square errors for Block-pulse and continuous approximations of Example 1.

\begin{tabular}{l|cc}
\hline Error & $m=20$ & $m=40$ \\
\hline Block pulse & $0.69 \times 10^{-4}$ & $0.17 \times 10^{-4}$ \\
CA & $0.46 \times 10^{-9}$ & $0.29 \times 10^{-10}$ \\
\hline
\end{tabular}

approximations are shown. The following is used for least square errors for the approximation $\bar{y}(t)$ of $y(t)$ :

$$
\int_{0}^{1}(y(t)-\bar{y}(t))^{2} d t=\frac{1}{m} \sum_{i=1}^{m} \int_{(i-1) / m}^{i / m}\left(y\left(\tau_{i}\right)-\bar{y}\left(\tau_{i}\right)\right)^{2} d \tau_{i} .
$$

4.2. Example 2. Consider the equation

$$
y(t)=1+\sin ^{2}(t)-3 \int_{0}^{t} \sin (t-s) y^{2}(s) d s, \quad t \in[0,1) .
$$

By using the method in Section 4, (4.3) is solved. The computational results for DA and CA of $y(t)$ with $m=60$ and $m=80$, together with the exact solution $y(t)=\cos t$, are given in Table 4.3. In Table 4.4, the least square errors for Block-pulse and continuous approximations are shown.

\section{Conclusion}

The properties of STWS are used to solve the nonlinear Volterra-Hammerstein integral equations. The problem has been reduced to solving a system of nonlinear algebraic equations. Illustrative examples are included to demonstrate the validity and applicability of 
Table 4.3. Estimated values of $y(t)$ using STWS with $m=60$ and 80 together with exact values of Example 2 .

\begin{tabular}{|c|c|c|c|c|c|}
\hline \multicolumn{2}{|c|}{$t$ DA with $m=60$} & \multirow{2}{*}{$\begin{array}{c}\text { CA with } m=60 \\
1.00000\end{array}$} & \multirow{2}{*}{$\begin{array}{c}\text { DA with } m=80 \\
1.00000\end{array}$} & \multirow{2}{*}{$\begin{array}{c}\text { CA with } m=80 \\
1.00000\end{array}$} & \multirow{2}{*}{$\begin{array}{c}\text { Exact } \\
1.00000\end{array}$} \\
\hline 0.0 & 1.00000 & & & & \\
\hline 0.1 & 0.99500 & 0.99500 & 0.99500 & 0.99500 & 0.99500 \\
\hline 0.2 & 0.98006 & 0.98006 & 0.98006 & 0.98006 & 0.98007 \\
\hline 0.3 & 0.95531 & 0.95532 & 0.95533 & 0.95533 & 0.95534 \\
\hline 0.4 & 0.92102 & 0.92103 & 0.92104 & 0.92105 & 0.92106 \\
\hline 0.5 & 0.87752 & 0.87754 & 0.87756 & 0.87756 & 0.87758 \\
\hline 0.6 & 0.82528 & 0.82529 & 0.82530 & 0.82531 & 0.82533 \\
\hline 0.7 & 0.76478 & 0.76480 & 0.76481 & 0.76482 & 0.76484 \\
\hline 0.8 & 0.69663 & 0.69665 & 0.69667 & 0.69669 & 0.69671 \\
\hline 0.9 & 0.62152 & 0.62156 & 0.62158 & 0.62159 & 0.62161 \\
\hline 0.1 & 0.54022 & 0.54025 & 0.54027 & 0.54028 & 0.54030 \\
\hline
\end{tabular}

Table 4.4. Least square errors for Block-pulse and continuous approximations of Example 2.

\begin{tabular}{l|cc}
\hline Error & $m=60$ & $m=80$ \\
\hline Block pulse & $0.36 \times 10^{-5}$ & $0.16 \times 10^{-5}$ \\
CA & $0.25 \times 10^{-8}$ & $0.50 \times 10^{-9}$ \\
\hline
\end{tabular}

the technique. By using the STWS direct method we can obtain pointwise, Block-pulse, and continuous approximations for the solution of $y$ in (1.1). Furthermore, the method does not require operational matrices of integration and unlike the Walsh series approach the method does not require the Kronecker product or the inversion of large matrices and there is no restriction on $m$ as in the case with Walsh series approach.

\section{References}

[1] K. Balachandran and K. Murugesan, Analysis of nonlinear singular systems via STWS method, Int. J. Comput. Math. 36 (1990), no. 1-2, 9-12.

[2] _ Numerical solution of a singular non-linear system from fluid dynamics, Int. J. Comput. Math. 38 (1991), no. 3-4, 211-218.

[3] H. Brunner, Implicitly linear collocation methods for nonlinear Volterra equations, Appl. Numer. Math. 9 (1992), no. 3-5, 235-247.

[4] R. Y. Chang and M. L. Wang, Solutions of integral equations via shifted Legendre polynomials, Internat. J. Systems Sci. 16 (1985), no. 2, 197-208.

[5] J. H. Chou and I. R. Horng, Double-shifted Chebyshev series for convolution integral and integral equations, Internat. J. Control 42 (1985), no. 1, 225-232.

[6] G. Q. Han, Asymptotic error expansion of a collocation-type method for Volterra-Hammerstein integral equations, Appl. Numer. Math. 13 (1993), no. 5, 357-369.

[7] C. H. Hsiao and C. F. Chen, Solving integral equations via Walsh functions, Comput. Electr. Engrg. 6 (1979), no. 4, 279-292. 


\section{Nonlinear integral equations via STWS}

[8] C. Hwang and Y. P. Shih, Solution of integral equations via Laguerre polynomials, Comput. Electr. Engrg. 9 (1982), 123-129.

[9] K. Kumar and I. H. Sloan, A new collocation-type method for Hammerstein integral equations, Math. Comp. 48 (1987), no. 178, 585-593.

[10] P. Lancaster, Theory of Matrices, Academic Press, New York, 1969.

[11] L. J. Lardy, A variation of Nyström's method for Hammerstein equations, J. Integral Equations 3 (1981), no. 1, 43-60.

[12] G. P. Rao, K. R. Palanisamy, and T. Srinivasan, Extension of computation beyond the limit of initial normal interval in Walsh series analysis of dynamical systems, IEEE Trans. Automat. Control 25 (1980), no. 2, 317-319.

[13] M. Razzaghi and J. Nazarzadeh, Walsh functions, Wiley Encyclopedia of Electrical and Electronics Engineering 23 (1999), 429-440.

[14] M. Razzaghi, M. Razzaghi, and A. Arabshahi, Solutions of convolution integral and Fredholm integral equations via double Fourier series, Appl. Math. Comput. 40 (1990), no. 3, 215-224.

[15] P. Sannuti, Analysis and synthesis of dynamic systems via block-pulse functions, Proc. IEE Control Applic. 124 (1977), no. 6, 569-571.

[16] F. G. Tricomi, Integral Equations, Dover Publications, New York, 1985.

[17] S. Yalçinbaş, Taylor polynomial solutions of nonlinear Volterra-Fredholm integral equations, Appl. Math. Comput. 127 (2002), no. 2-3, 195-206.

B. Sepehrian: Department of Mathematics, Arak University, Tehran, Iran

E-mail address: s7713971@cic.aku.ac.ir

M. Razzaghi: Department of Mathematics and Statistics, Mississippi State University, MS 39762, USA; Department of Applied Mathematics, Amirkabir University of Technology, Tehran 15914, Iran

E-mail address: razzaghi@math.msstate.edu 


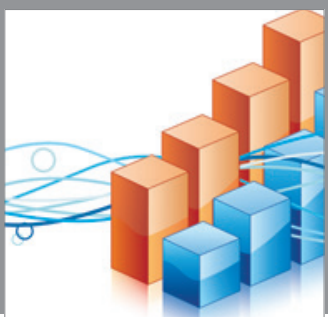

Advances in

Operations Research

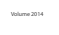

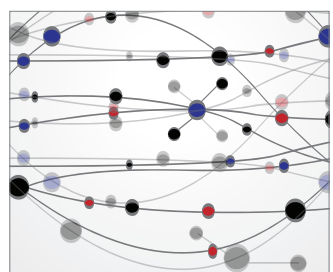

\section{The Scientific} World Journal
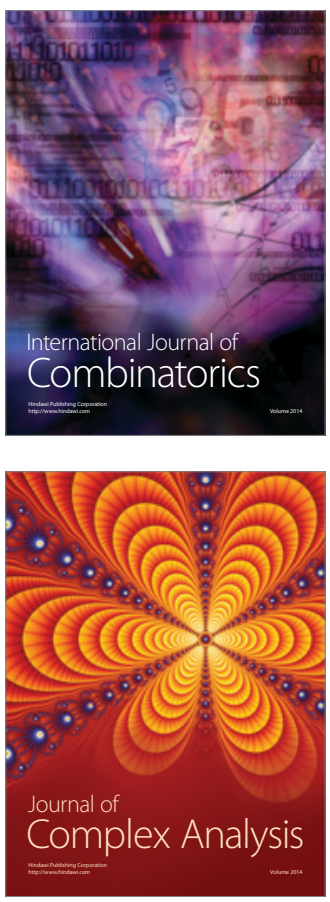

International Journal of

Mathematics and

Mathematical

Sciences
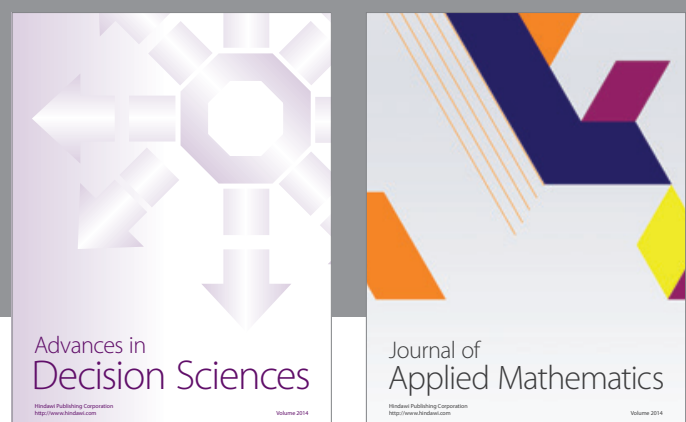

Journal of

Applied Mathematics
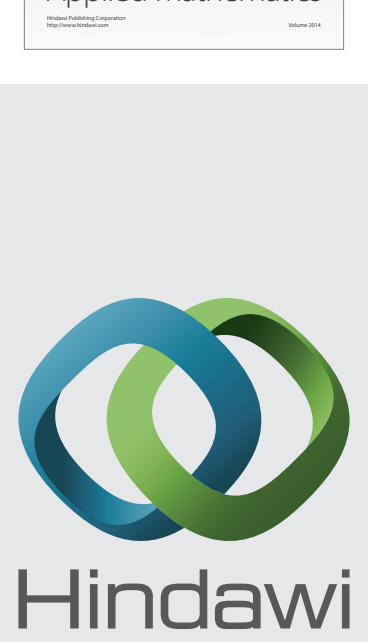

Submit your manuscripts at http://www.hindawi.com
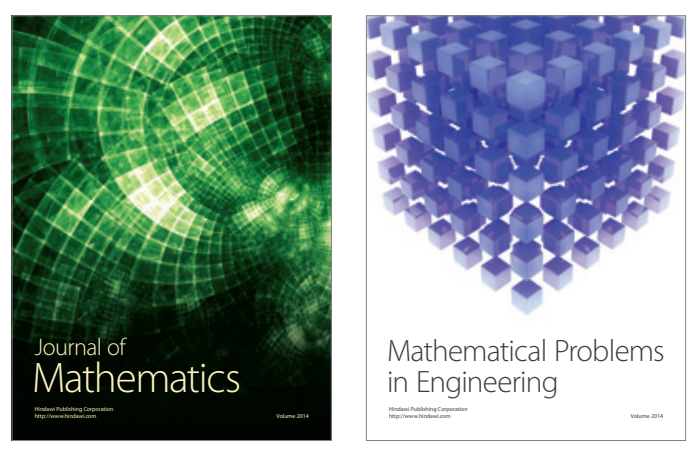

Mathematical Problems in Engineering
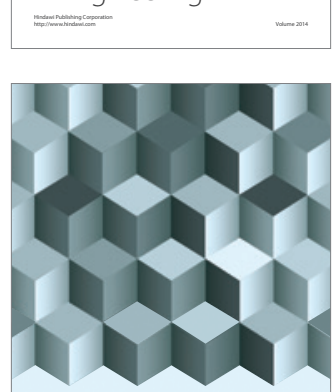

Journal of

Function Spaces
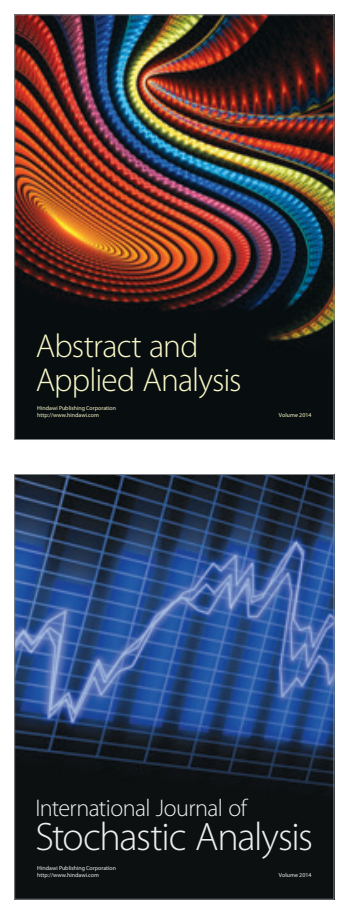

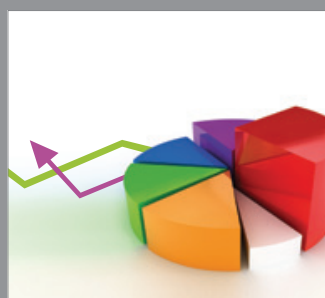

ournal of

Probability and Statistics

Promensencen
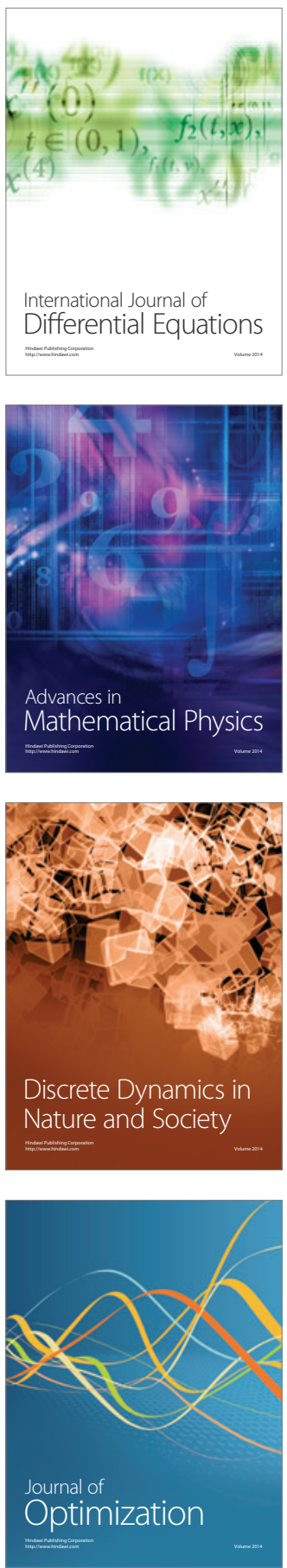